\title{
Vibration Response Characteristics against the Radial and Axial Shocks on Small Size Hard Disk Drive Spindle Supported by Oil Film Bearings
}

\author{
Masayuki Ochiai, Yuta Sunami and Hiromu Hashimoto \\ Department of Mechanical Engineering, Tokai University, Kanagawa 259-1292, Japan
}

Received: March 20, 2014 / Accepted: April 08, 2014 / Published: July 25, 2014.

\begin{abstract}
This paper describes the experimental study on shock response of FDB (fluid dynamic bearing) spindle for HDDs (hard disk drives). The FDBs are widely used as rotating shaft support elements for HDD spindle motors. Recently, the opportunity for the HDD spindle motors exposed to external vibration has been increasing because the HDDs are used for various information related equipment such as mobile PCs (personal computers), video cameras, car navigation systems and so on. Hence, the rotating shaft has a possibility to come in contact with the bearing by external shocks and it causes wear or seizure to the bearing surface. To avoid the problem, it is extremely important to know how the spindle moves against the large shock on HDDs experimentally. However, as far as the authors know, there are few experimental studies treating the shock response of HDD spindles. In this paper, firstly, we propose a new test rig and experimental method for shock response of FDB spindles. Then the shock tests against the radial and axial disturbance on FDB spindle for 2.5" HDD are conducted. Finally, the experimental results of shock response waveforms and maximum displacement of disk are shown.
\end{abstract}

Key words: Tribology, fluid film bearing, hard disk drive, spindle motor, shock test, shock response.

\section{Introduction}

The oil film bearings are widely used as rotating shaft support elements for HDDs (hard disk drives) spindle motors. Recently, the opportunity for the HDD spindle motors exposed to external vibration has been increasing because the HDDs are used for various information related equipment such as mobile PCs (personal computers), video cameras, car navigation systems and so on. Hence, the rotating shaft has a possibility to come in contact with the bearing by the external shocks and it causes wear or seizure to the bearing surface. In order to avoid the problems, it is extremely important to know how the spindle moves against the large shock on HDDs experimentally.

Under the background, much research about the

Corresponding author: Masayuki Ochiai, Ph. D., research fields: mechanical engineering, tribology, optimum design, and machine element. E-mail: ochiaim@keyaki.cc.u-tokai.ac.jp. dynamic characteristics of HDD spindles has been studied [1-5]. However, as far as the authors know, there are few experimental studies treating the response against the shocks. The reason why there is few experimental research concerning about the shock response is difficulty of measurement. Because, the falling type tests, which generally used, have high possibility to break sensors. Consequently, usual experimental research regarding the shocks has been studied whether the HDDs subjected to external shocks break or not.

On the other hand, Ku et al. [6] measured the shock response of HDD spindle against the radial shocks by using the shaker. However, in this study, only one degree response in the radial shock direction was measured and the conical mode vibration of the spindle was not measured. If the conical mode vibrations occur in HDD spindle operation, there is high possibility to come in contact with bearing 
surface which causes wear or seizure of bearings. Therefore, it is very important to measure the conical mode responses when we evaluate the durability or reliability of HDD spindles.

At the same time, Tohma et al. [7] and Wu et al. [8] measured the conical mode vibrations. However, they investigated the frequency characteristics of spindle motor. Consequently, they only gave the small shocks to the spindles and they did not study about the possibility of contact and the durability of spindles.

The paper is organized as follows: Section 2 presents a new experimental equipment; Section 3 presents a new test method; Section 4 shows the test results of the shock response of HDD spindles specifically and discussion; and Section 5 gives the conclusions.

\section{Test Spindle Motor and Test Rig}

\subsection{Test Spindle Motor}

Fig. 1 shows the schematic diagram of the typical spindle motor for 2.5" HDDs. The rotor part of the spindle which consists of a disk, a hub and a shaft is supported by upper and lower journal bearings and thrust bearings. In the actual HDD spindle design, it is difficult to put in the same position of the center of gravity of the rotor and the moment center of the journal bearings because of the limitation of the HDD structure. Consequently, the vibration mode of spindle becomes not only parallel mode but also conical mode when the radial shocks act on the spindle and we should study the effects of both vibration mode experimentally. Fig. 2 shows the geometry of journal and thrust bearings and Table 1 shows the specifications of fluid bearing spindle using in this experiment.

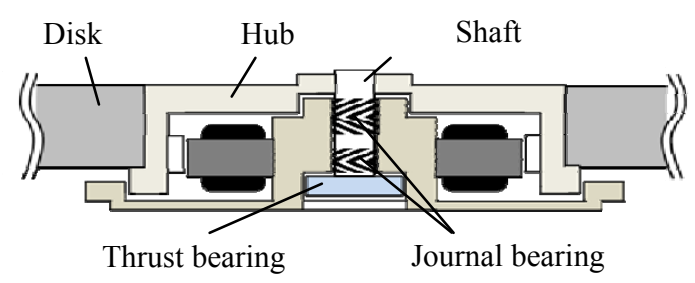

Fig. 1 Schematic diagram of spindle motor for 2.5 ” HDD.

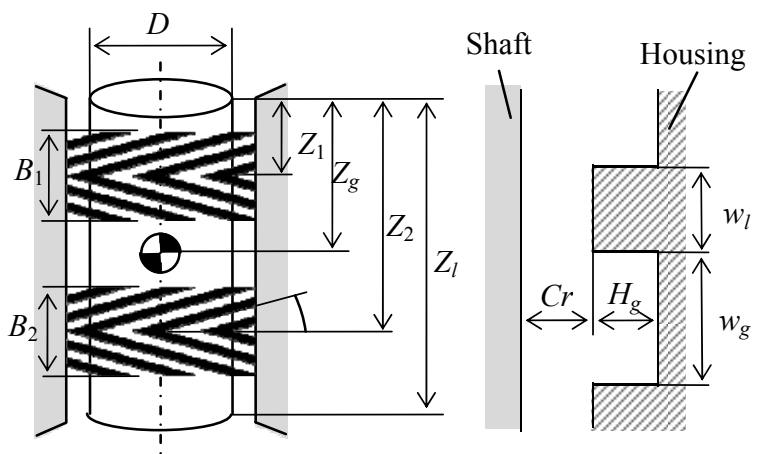

(a) Journal bearing

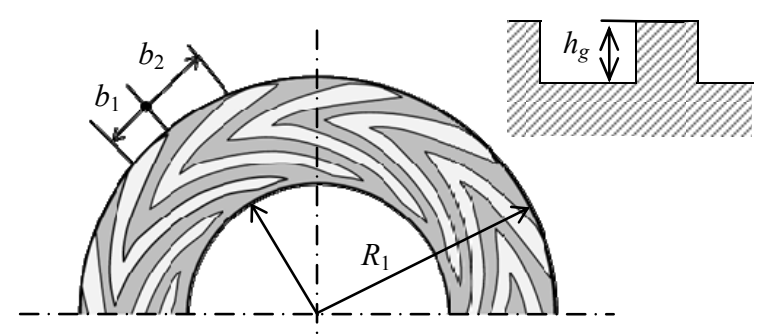

(b) Thrust bearing

Fig. 2 Geometry of journal and thrust bearings.

\subsection{Test Rig for Shock Responses}

Generally, falling type test rigs are used for HDD shock tests [9], however using these types of test rigs there are high possibility to break the test instruments for example the displacement sensors for measuring the spindle response. Hence, we propose another type of shock test rig as shown in Fig. 3. The test rig consists of the spindle motor, a motor fixing jig, eddy-current proximity proves, an acceleration pickup, four poles, a surface plate and a pendulum. Using the pendulum mechanism, we can give the shocks to the test spindles with reducing the risk of breaking the sensors. Furthermore, we provide the sliding mechanism using the oval halls as shown in Fig. 3b for giving the half-sine shocks to the test spindle. At the top of the pendulum, there is an interchangeable chip made of hard rubber. Changing the chip material allows us to adjust the shock duration.

Fig. 4 shows the typical acceleration wave forms measured with the acceleration pickup. In Fig. 4, it is confirmed that half-sin waveforms obtained 
Table 1 Bearing specifications.

\begin{tabular}{|c|c|c|c|}
\hline & Parameters & & Values \\
\hline \multirow{12}{*}{ Journal bearing } & Number of grooves & $N_{j}$ & 14 \\
\hline & Bearing diameter & $D(\mathrm{~mm})$ & 3.2 \\
\hline & Radius clearance & $C r(\mu \mathrm{m})$ & 3.0 \\
\hline & Bearing length & $Z_{l}(\mathrm{~mm})$ & 4.2 \\
\hline & Bearing position 1 & $Z_{1}(\mathrm{~mm})$ & 1.2 \\
\hline & Bearing position 2 & $Z_{2}(\mathrm{~mm})$ & 3.2 \\
\hline & Bearing width 1 & $B_{1}(\mathrm{~mm})$ & 1.6 \\
\hline & Bearing width 2 & $B_{2}(\mathrm{~mm})$ & 1.2 \\
\hline & Groove depth & $H_{g}(\mu \mathrm{m})$ & 5.0 \\
\hline & Groove angle & $\beta(\operatorname{deg})$ & 25 \\
\hline & Groove and land ratio & $\alpha=\left(w_{1} /\left(w_{1}+w_{2}\right)\right)$ & 0.5 \\
\hline & Oil viscosity $\left(30^{\circ} \mathrm{C}\right)$ & $\mu(\mathrm{Pa} \cdot \mathrm{s})$ & $1.18 \times 10^{-2}$ \\
\hline \multirow{6}{*}{ Thrust bearing } & Number of grooves & $N_{t}$ & 12 \\
\hline & Outer radius & $R_{1}(\mathrm{~mm})$ & 2.7 \\
\hline & Inner radius & $R_{2}(\mathrm{~mm})$ & 1.5 \\
\hline & Groove depth & $h_{g}(\mu \mathrm{m})$ & 12 \\
\hline & Groove width ratio & $\gamma=\left(b_{1} /\left(b_{1}+b_{2}\right)\right)$ & 0.51 \\
\hline & Oil viscosity $\left(30^{\circ} \mathrm{C}\right)$ & $\mu(\mathrm{Pa} \cdot \mathrm{s})$ & $1.18 \times 10^{-2}$ \\
\hline \multirow{4}{*}{ Rotor } & Center of gravity of rotor & $Z_{g}(\mathrm{~mm})$ & 0.8 \\
\hline & Rotor mass & $m(\mathrm{~g})$ & 18.8 \\
\hline & Inertia moment of rotor & $I\left(\mathrm{~kg} \cdot \mathrm{m}^{2}\right)$ & $1.06 \times 10^{-6}$ \\
\hline & Polar inertia moment of rotor & $I_{p}\left(\mathrm{~kg} \cdot \mathrm{m}^{2}\right)$ & $2.04 \times 10^{-6}$ \\
\hline
\end{tabular}

and the shock durations can be controlled within the value of the maximum acceleration of $20 \mathrm{G}$.

Fig. 5 shows the test rig for the axial shock response. In this experimental study, we can measure the axial shock response by changing the combination of the test rig parts. The shock forces are given from bottom of the motor fixing jig.

\section{Measurement Method}

Fig. 6 shows the geometry of test disk. Four directional displacements of disk supported by oil film bearings are measured using the eddy-current type sensors. The axial displacements of $\Delta Z_{x}$ and $\Delta Z_{y}$ are measured at the position of $\mathrm{A}$ and $\mathrm{B}$ on the disk surface and the radial displacements of $\Delta x$ and $\Delta y$ are measured at the position of $\mathrm{A}^{\prime}$ and $\mathrm{B}^{\prime}$ on the disk end face as shown in Fig. 6. Consequently, we can measure the conical mode vibration of spindle with parallel mode vibration from these displacement data when the radial shocks are given. Table 2 shows the specifications of the disk.

Lissajous waveforms for each positions of the shaft, as shown in Fig. 6, are calculated by the formula as follows.

$$
\left\{\begin{array}{l}
x_{U}=\Delta x+Z_{g} \sin \theta_{x} \\
y_{U}=\Delta y+Z_{g} \sin \theta_{y} \\
x_{L}=\Delta x-\left(Z_{l}-Z_{g}\right) \sin \theta_{x} \\
y_{L}=\Delta y-\left(Z_{l}-Z_{g}\right) \sin \theta_{y}
\end{array}\right.
$$

where,

$$
\left\{\begin{array}{l}
\theta_{x}=\tan ^{-1}\left(\Delta_{x} / r\right) \\
\theta_{y}=\tan ^{-1}\left(\Delta_{y} / r\right)
\end{array}\right.
$$

On the other hand, the axial responses were measured by using the two sensors at the positions of axial displacement of $\Delta Z_{x}$ and $\Delta Z_{y}$, each of which is 

Spindle Supported by Oil Film Bearings

basically same value because the axial response becomes parallel mode vibration.

The measurement conditions of rotational speed were 4,200, 5,400 and 7,200 rpm, the acceleration of shocks were $5 \mathrm{G}$ to $20 \mathrm{G}$ every $5 \mathrm{G}$, and shock durations were $3.0 \mathrm{~ms}$.

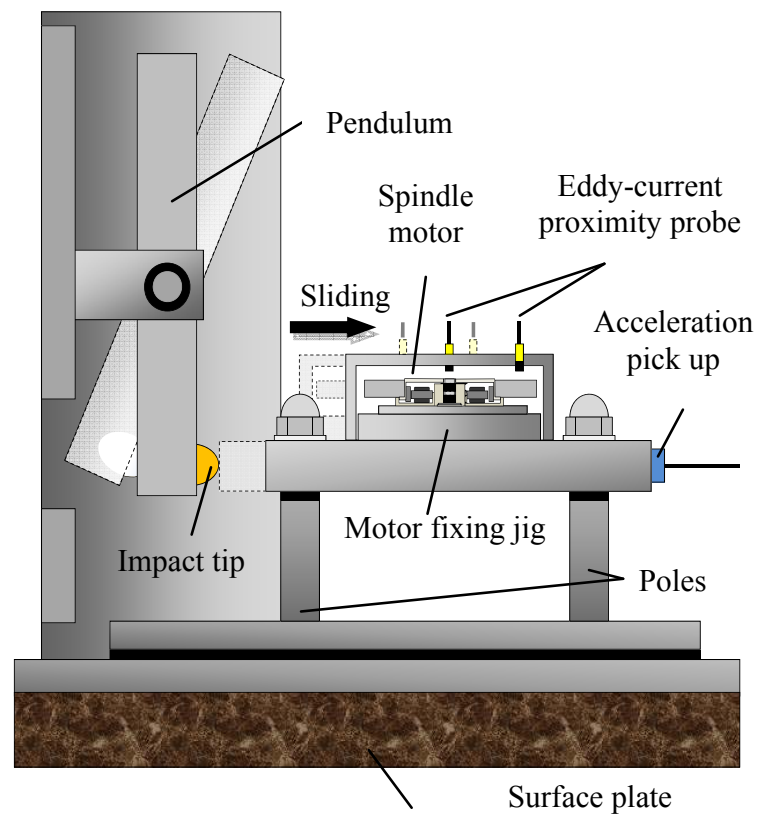

(a) Overview of test rig

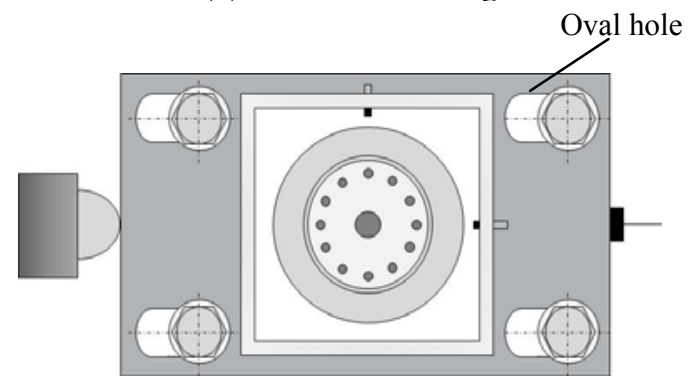

(b) Close up from a top view

Fig. 3 Radial impact test rig.

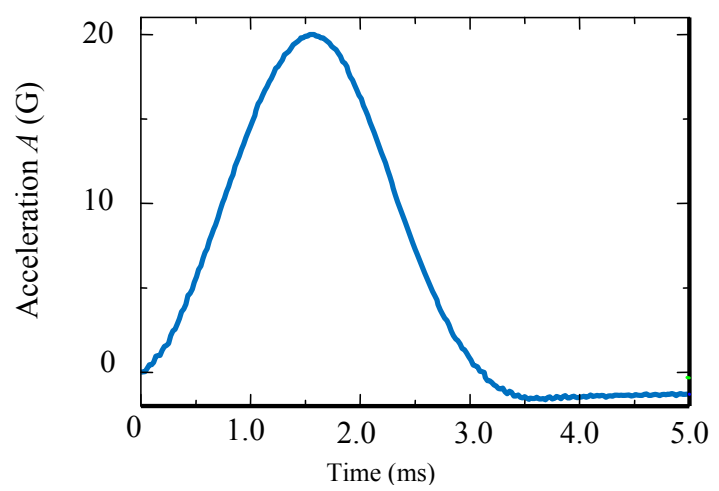

Fig. 4 Shock acceleration waveforms.

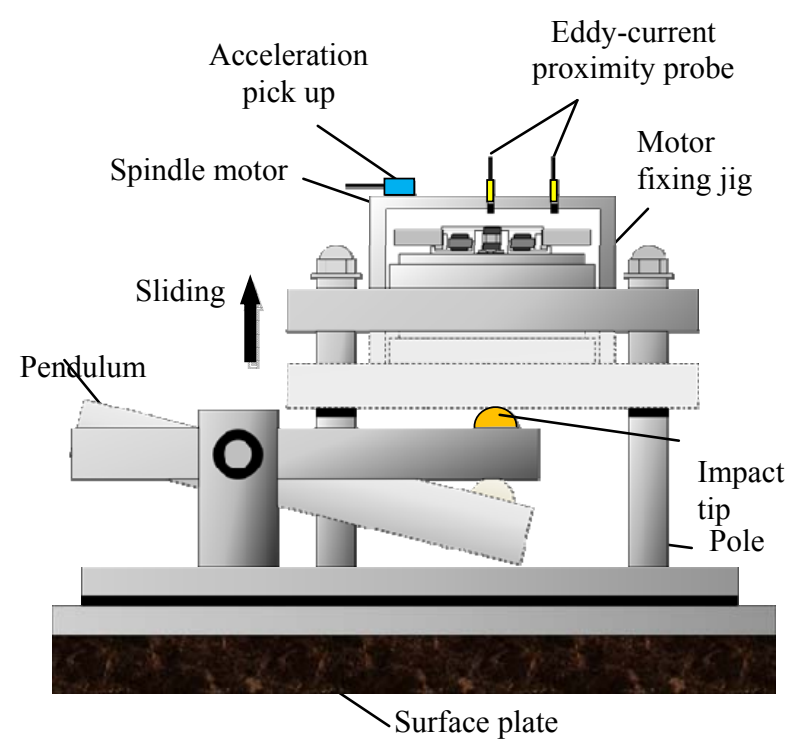

Fig. 5 Axial shock test rig.

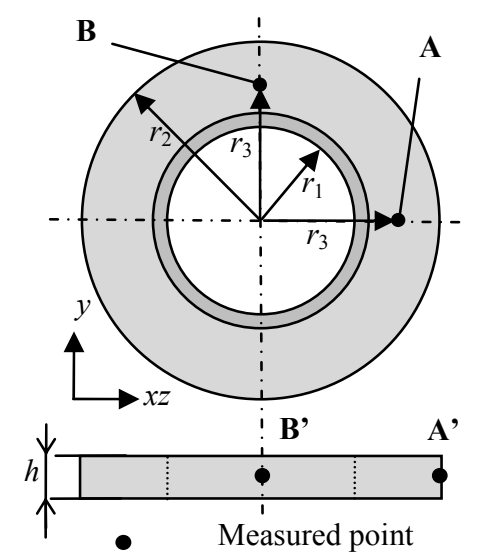

Fig. 6 Detail of disk.

Table 2 Specifications of disk.

\begin{tabular}{ll}
\hline Material & SUS304 \\
\hline Inner radius $r_{1}(\mathrm{~mm})$ & 10.0 \\
Outer radius $r_{2}(\mathrm{~mm})$ & 19.3 \\
Thickness $h(\mathrm{~mm})$ & 3.50 \\
Measured position $r_{3}(\mathrm{~mm})$ & 14.0 \\
\hline
\end{tabular}

In addition, the measured displacement waveforms with the eddy current sensors contain the vibration component of RRO (repeatable run out). Hence, in this study we subtract the RRO components, which measured preliminary experiment, from measured displacement waveforms. We can obtain the displacement waveforms of shock response after that data processing. 


\section{Experimental Results and Discussion}

\subsection{Radial Shock Response}

Figs. $7 \mathrm{a}$ and $7 \mathrm{~b}$ show the experimental results of the radial displacement waveforms of $\Delta x$ and $\Delta y$ against the time under the condition of radial shock acceleration of $20 \mathrm{G}$. In Fig. 7, the solid lines indicate the rotational speed of 4,200 rpm and the single dotted lines and broken lines indicate 5,400 rpm and 7,200 rpm, respectively.

As shown in Fig. 7a, the displacements $\Delta x$ firstly rise up after the radial shocks and then converged rapidly caused by damping effect of bearings oil film. On the other hand, as shown in Fig. 7b, the displacements $\Delta y$ also rise up in spite of perpendicular to shock direction and then converged. It is due to coupled effect of oil film bearing. In addition, it is

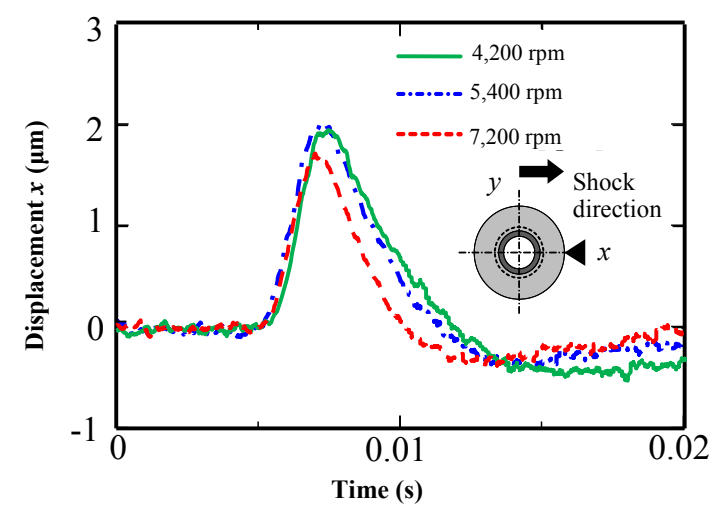

(a) $x$ direction found that the value of the maximum displacements of $\Delta y$ are about half of maximum ones of $\Delta x$.

Figs. $8 \mathrm{a}$ and $8 \mathrm{~b}$ show the experimental results of the axial displacement wave forms of $\Delta Z_{x}$, and $\Delta Z_{y}$ with time under the conditions of radial shock acceleration of $20 \mathrm{G}$. In this radial shock tests, the axial response waveforms mean the displacement caused by conical mode vibrations.

From the results of the axial displacement $\Delta Z_{x}$, as shown in Fig. 8a, the disk inclines caused by radial shock and the response damps rapidly. In addition, as shown in Fig. 8b, the displacement of $\Delta Z_{y}$ also appeared caused by radial shock.

Here focusing on the influence of the rotational speeds, the displacement of $\Delta Z_{x}$ decreases with increasing the rotational speed. It is due to the increase of bearing stiffness of journal bearings. On the other

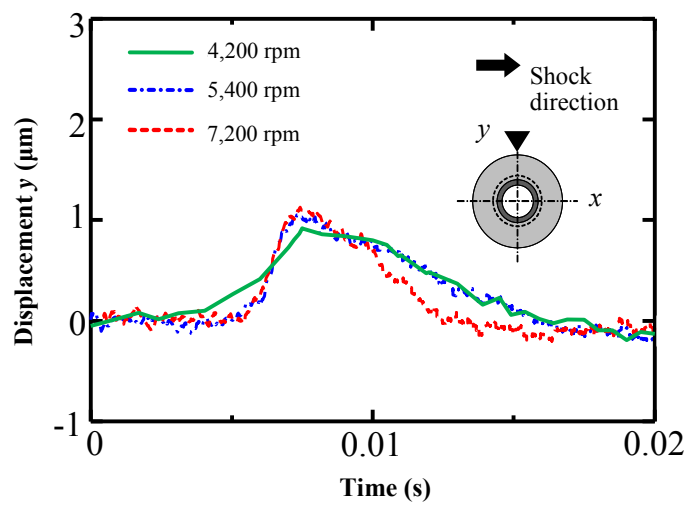

(b) $y$ direction

Fig. 7 Response displacement waveform of radial direction (radial shock acceleration 20 G, shock duration 3 ms)

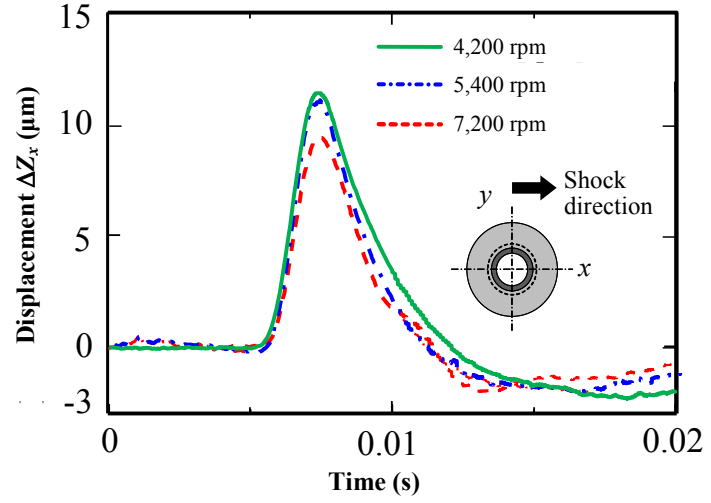

(a) $x$ direction

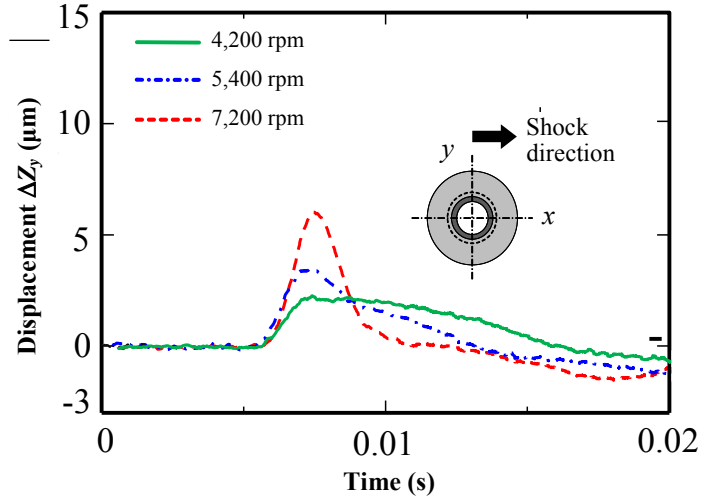

(b) $y$ direction

Fig. 8 Response displacement waveform of axial direction (radial shock acceleration $20 \mathrm{G}$, shock duration 3 ms), response displacement of axial direction ( measured five times ) 


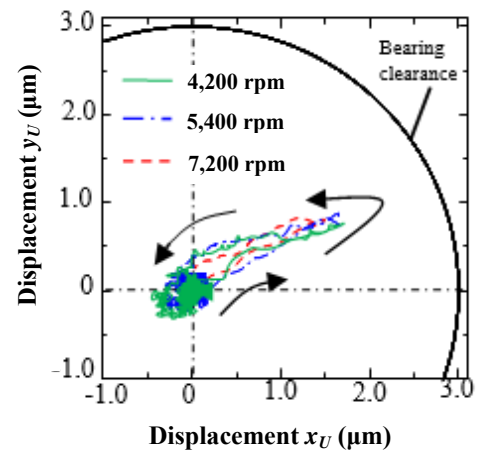

(i) $10 \mathrm{G}$

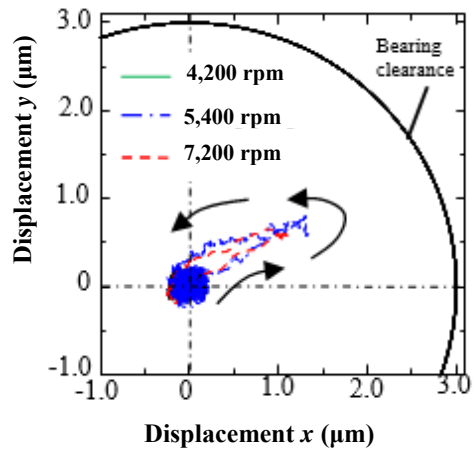

(i) $10 \mathrm{G}$

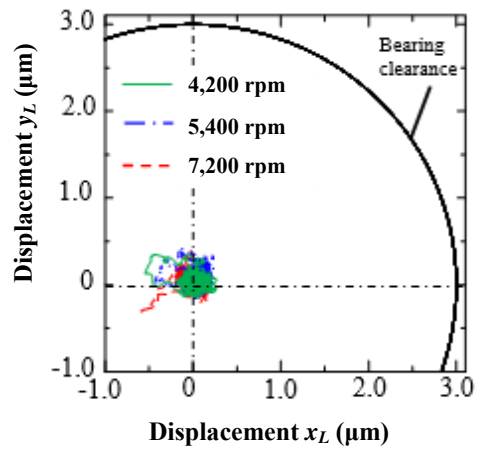

(i) $10 \mathrm{G}$

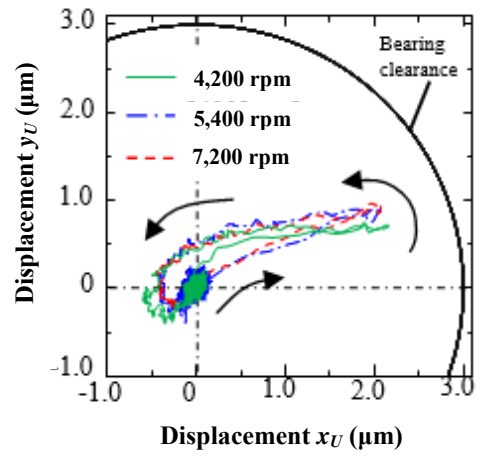

(ii) $15 \mathrm{G}$

(a) Position of upper end

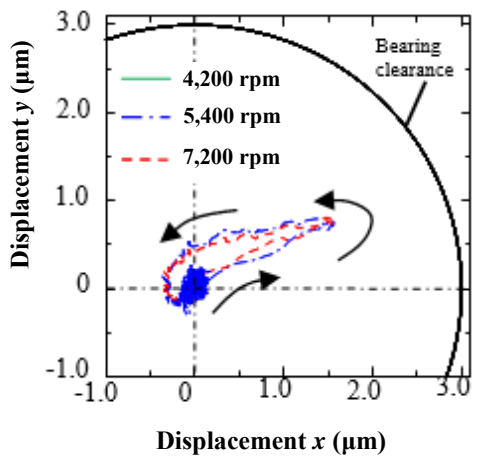

(ii) $15 \mathrm{G}$

(b) Position of center of gravity

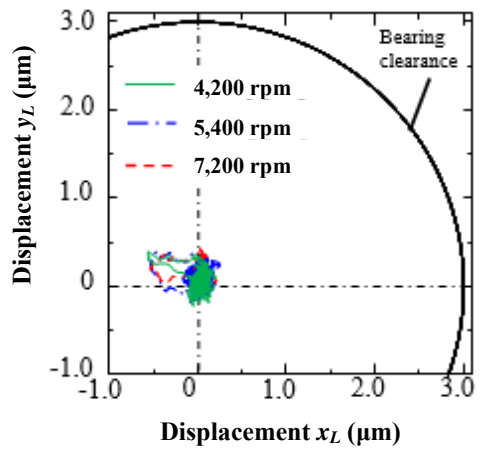

(ii) 15 G

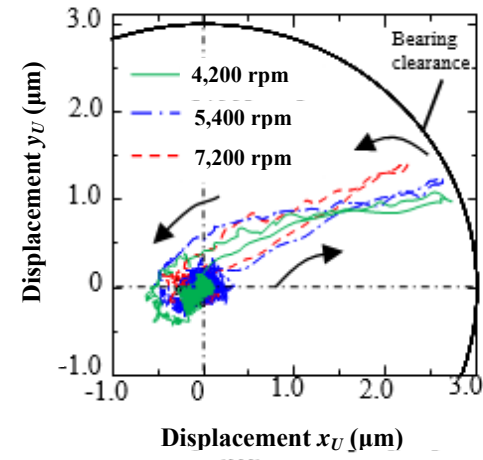

(iii) $20 \mathrm{G}$

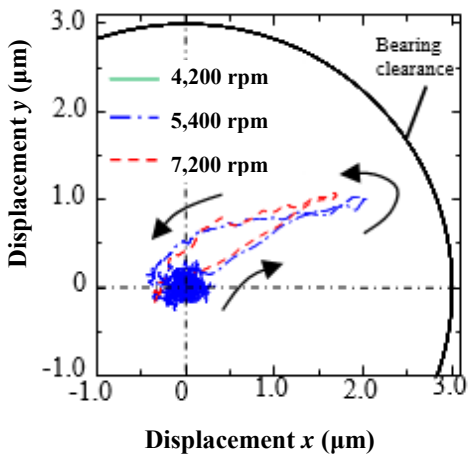

(iii) $20 \mathrm{G}$

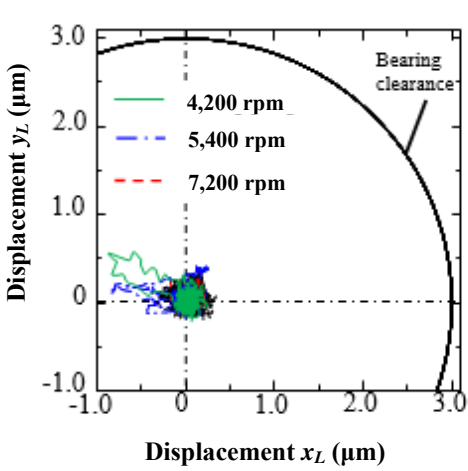

(iii) $20 \mathrm{G}$

(c) Position of lower end

Fig. 9 Lissajous waveforms of shaft at each bearing position.

hand, the displacement of $\Delta Z_{y}$ decreases significantly with increasing of the rotational speed. The discrepancy can be explained by gyro effect and coupling effect of oil film stiffness. Because the gyro moment and the coupling component of the oil film stiffness increases with increasing the rotational speed.
Fig. 9 shows the lissajous waveforms at the position of (a) upper end of the bearing sleeve, (b) center of gravity of rotor and (c) the lower end of the bearing sleeve. In addition, the experiments are conducted under conditions of the radial shock acceleration of 10 $\mathrm{G}$ to $20 \mathrm{G}$, the shock duration of $3 \mathrm{~ms}$. In Fig. 9, solid lines, single dotted lines and broken lines indicate the 
rotational speed of $4,200,5,400$ and $7,200 \mathrm{rpm}$, respectively.

As shown in Figs. 9a and 9b, in the positions of upper end and the center of gravity, the shaft moves firstly approximately to the direction of 30 degree against the horizontal line over the whole accelerations and rotational speeds. The shaft center comes buck to bearing center within arched shape after the maximum values of displacement. After that, the vibrations are converged rapidly. This is due to the short delay of displacement of $y$ direction which is perpendicular to shock direction. Because the displacements of $y$ occurs caused by coupling effect of bearing stiffness.

It is found that the largest displacements are obtained at the upper end of the bearing sleeve in whole three positions. On the other hand, the displacements at the lower end position almost remain the central position. From these results, the shaft vibrates in conical mode within the pivot of near the lower end of bearing sleeve. Therefore, it is considered that there is high possibility to come in contact with the bearing surface and shaft at the upper end of the bearing sleeve.

In addition, it is found that the trajectories of each rotational speeds under $20 \mathrm{G}$ are little different from other acceleration trajectories. This is due to gyroscopic effect. Because the gyroscopic moment increase caused by increasing of inclination of shaft reads to trajectory difference. Especially, the effect at the high speed 7,200 rpm becomes remarkable.

From the results shown in Fig. 9, it is confirmed that there is no contact of shaft and bearing surfaces under this experimental conditions.

\subsection{Axial Shock Response}

Fig. 10 shows the response displacement waveform against the axial shock under the axial acceleration of $20 \mathrm{G}$, the shock duration of $3 \mathrm{~ms}$. It is found that the maximum value of the axial displacement decreases with an increase of rotational speed, because the bearing stiffness of the thrust bearing increases with increasing of rotational speed. There is no contact of the bearing surfaces, because the flying height of the thrust bearing is approximately $6 \mu \mathrm{m}$.

Fig. 11 shows the maximum displacement with axial shock acceleration. The maximum displacements increase almost linearly with increasing the axial shock acceleration. The maximum displacement decreases with an increase of rotational speed in whole acceleration conditions, because of increase of thrust bearing dynamic stiffness with increasing of rotational speed. In Fig. 11, it can be found that there is no contact of the bearing surfaces under these conditions.

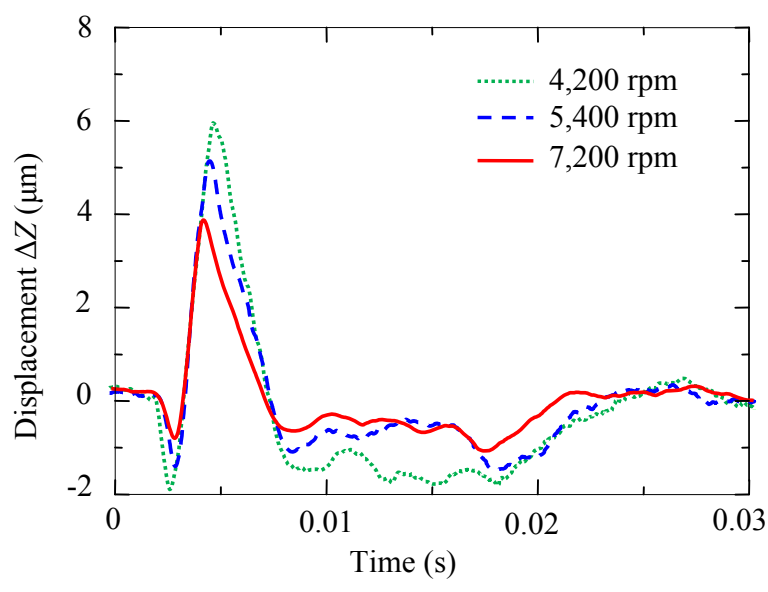

Fig. 10 Response displacement waveforms (axial acceleration 20 G, shock duration 3 ms).

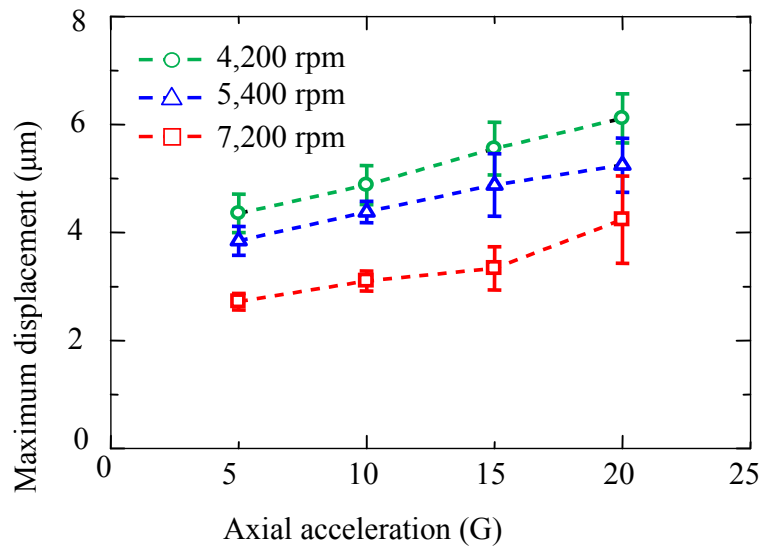

Fig. 11 Maximum displacement against axial shock responses (shock duration $3 \mathrm{~ms}$ ). 

Spindle Supported by Oil Film Bearings

\section{Conclusions}

In this paper, we described the vibration characteristics of small size HDD spindle against the radial and axial shocks. Main conclusions are shown as follows.

We proposed a new test rig using the pendulum construction and it was confirmed that we can give the half sinusoidal shocks to the test spindle by using the test rig.

From the results of the lissajous waveforms, it is found that the shaft works with the conical mode in which the pivot is near the lower end of the bearing sleeve.

The amplitudes of both radial and axial shock responses decrease with increasing the rotational speed.

It was found that there are no contact of the journal and the thrust bearings for 2.5 inch HDD spindle against the radial and the axial shocks under conditions of the shock acceleration of $20 \mathrm{G}$ and the shock durations $3.0 \mathrm{~ms}$.

\section{Acknowledgment}

A part of this research was supported by Grant-in-Aid for Scientific Research (C) (No. 25420098). The authors express their deep gratitude.

\section{References}

[1] Zhu, J., and Ono, K. 1999. "A Comparison Study on the
Performance of Four Types of Oil Lubricated Hydrodynamic Thrust Bearings for Hard Disk Spindles." Journal of Tribology ASME 121 (1): 114-21.

[2] Jang, G. H. 2005. "Dynamic Analysis of a HDD Spindle System with FDBs Due to the Bearing Width and Asymmetric Grooves of Journal Bearing." Journal of Microsystem Technologies 11 (7): 499-505.

[3] Jang, G. H., Lee, S. H., and Kim, H. W. 2006. "Finite Element Analysis on the Coupled Journal and Thrust Bearing in a Computer Hard Disk Drive." Journal of Tribology ASME 128 (2): 335-40.

[4] Miwa, M., Miyazaki, H., Kaneko, R., and Unozawa, H. 2005. "Evaluation of Fluid Dynamic Bearing Spindle by Vi-bration Base." IEEE Transactions on Magnetics 41 (2): 763-69.

[5] Ochiai, M., Sunami, Y., and Hashimoto, H. 2014. "Identification Method of Dynamic Coefficients of Fluid Film Bearings for HDD Spindle Motors." Journal of Mechanics Engineering and Automation 4 (2): 123-29.

[6] Ku, R., and Shumway, M. 1998. "Experimental Investigation of Shock Responses of Cantilever-Shaft Design Hydrodynamic Bearing Spindle Motors.” IEEE Transactions on Magnetics 34 (4): 1913-15.

[7] Tohma, S. 2007. "Frequency Analysis of Hard Disk Drive Spindle System Supported by Hydrodynamic Bearings, JSME Technical Journal." Journal of Advanced Mechanical Design, System, and Manufacturing 1 (5): 717-25.

[8] Wu, T. L. 2007. "Vibration of 1.8-in Hard Disk Drive Spindle Motors at Various Ambient Temperatures." IEEE Transactions on Magnetics 43 (9): 3716-20.

[9] Jung, K. M, and Jang, G. H. 2011. “Axial Shock-Induced Motion of the Air-Oil Interface of Fluid Dynamic Bearings of a non-Operating Hard Disk Drive." IEEE Transactions on Magnetics 47 (7): 1911-17. 